\title{
Shallow-water rogue waves: An approach based on complex solutions of the Korteweg-de Vries equation
}

\author{
A. Ankiewicz, Mahyar Bokaeeyan, and N. Akhmediev \\ Optical Sciences Group, Research School of Physics and Engineering, The Australian National University, Canberra, ACT 2600, Australia
}

(Received 1 February 2019; revised manuscript received 28 April 2019; published 30 May 2019)

\begin{abstract}
The formation of rogue waves in shallow water is presented in this Rapid Communication by providing the three lowest-order exact rational solutions to the Korteweg-de Vries (KdV) equation. They have been obtained from the modified KdV equation by using the complex Miura transformation. It is found that the amplitude amplification factor of such waves formed in shallow water is much larger than the amplitude amplification factor of those occurring in deep water. These solutions clearly demonstrate a potential hazard for coastal areas. They can also provide a solid mathematical basis for the existence of abnormally large-amplitude waves in other branches of nonlinear physics such as optics, unidirectional crystal growth, and in quantum mechanics.
\end{abstract}

DOI: 10.1103/PhysRevE.99.050201

\section{INTRODUCTION}

Rogue waves frequently hit beaches and coastal areas, causing significant damage and loss of life [1]. While significant efforts have been made to give explanations of rogue waves in the open ocean [2-5], little has been done about how to describe rogue waves that appear in coastal areas and shallow waters [1], although these constitute nearly $70 \%$ of the total number of extreme water wave events [6]. Rogue waves in the ocean are described by the nonlinear Schrödinger equation (NLSE) [7] and it has solutions in the form of rogue waves [8,9]. These phenomena have been observed in water tanks [10], thus confirming the mathematical description of these devastating phenomena.

In shallow water, the mathematical description of water waves is usually based on solutions of the Korteweg-de Vries $(\mathrm{KdV})$ or Kadomtsev-Petviashvili (KP) equations [1,11]. The $\mathrm{KdV}$ equation [12] is known to have soliton solutions [13,14] and these have been studied intensively, starting from the pioneering work of Zabusky and Kruskal [13]. In most of these works, the $\mathrm{KdV}$ equation is assumed to be real, i.e., the function $q(x, t)$ involved in this equation is taken to be real. This approximation is valid when the vertical velocities of the water particles are much smaller than the horizontal (along the free water surface) velocities. Levi [15] was the first to derive the complex function $\mathrm{KdV}$ for shallow-water waves. It takes into account the vertical components of the velocity. Clearly, in this case, a whole new world of complex solutions of the $\mathrm{KdV}$ equation have to be discovered and their relevance to shallow-water wave dynamics must be considered. In particular, rogue wave solutions, that were previously considered nonexistent for the $\mathrm{KdV}$ equation, must also be sought. The first attempts to find rogue wave solutions of the complex $\mathrm{KdV}$ equation were made in Ref. [16]. However, overall, little has been done on this subject, and our approach here is quite different from that of Ref. [16].

*ana124@physics.anu.edu.au
In this Rapid Communication, we overcome this deficiency by presenting rogue wave solutions of the complex function $\mathrm{KdV}$ equation. Indeed, the latter provides a more detailed description of waves in shallow water, as has been demonstrated by Levi $[15,17]$. We note that there is a close connection between the KdV and NLS equations [18,19]. Approximations of the NLSE by the KdV [20], or, inversely, the KdV by the NLSE [21,22], are frequently presented. However, the difficulty of using such approximations lies in the fact that they relate a complex function of the NLSE to a real function of the $\mathrm{KdV}$ equation. Then, this transform loses a significant amount of information that is contained in the NLSE. Retaining the complexity of the function in the $\mathrm{KdV}$ equation provides a more detailed description of waves than that obtained from the purely real function $\mathrm{KdV}$ equation. As a result, it allows us to find rogue wave solutions that otherwise would be "lost."

Another equation connected to the $\mathrm{KdV}$ is the modified $\mathrm{KdV}$ (i.e., mKdV). Although it has a similar mathematical structure, the mKdV does not directly describe shallow-water waves. Importantly, the latter has been shown recently [23] to have rogue wave solutions. In particular, it was shown that many features of $\mathrm{mKdV}$ rogue waves are similar to those of rogue waves described by the NLSE solutions [23]. Namely, the hierarchies of rogue wave solutions in each case, $\mathrm{mKdV}$ and NLSE, have the same amplitudes that increase with the order of the solution. These observations lead us to the conclusion that the complex function $\mathrm{KdV}$ equation may also have rogue wave solutions.

Indeed, it is well known that all solutions of the $\mathrm{KdV}$ and $\mathrm{mKdV}$ equations are related through the Miura transformation [24,25]. However, applying this transformation to the rogue wave solutions found in Ref. [23] leads to solutions that are singular and hence not physical. Thus, it is not the standard transformation [24,25] that has to be used in this case. In fact, there is a so-called complex Mirua transformation that has been used in finding complex solutions of the $\mathrm{KdV}$ from the real solutions of the $\mathrm{mKdV}$ [26]. It has been shown, by using this transformation, that soliton solutions of the 
focusing $\mathrm{mKdV}$ can be mapped to complex soliton solutions of the KdV equation. However, this transformation cannot be used to convert real soliton solutions of the defocusing $\mathrm{mKdV}$ to solutions for the complex function $\mathrm{KdV}$ equation. Surprisingly, for rational solutions, it is a completely different matter. Here, we will show that rational solutions of the $\mathrm{mKdV}$ equation can be mapped to complex rational solutions of the $\mathrm{KdV}$ equation using the complex Mirua transformation.

The KdV equation has a variety of complex solutions, including solitons $[27,28]$. In this Rapid Communication, we provide rogue wave solutions. Similar to the cases of NLSE and $\mathrm{mKdV}$, these are rational solutions that have maximum amplitude in the center and decay to the background both in time and in space, thus satisfying the mathematical definition of rogue waves suggested in Ref. [8].

We start with the $\mathrm{mKdV}$ equation,

$$
p_{x}+\beta p^{2} p_{t}-\gamma_{3} p_{t t t}=0,
$$

where $x$ is the evolution variable (time), $t$ is the spatial variable, and $p(x, t)$ is a real function describing the wave form. The values $\beta$ and $\gamma_{3}$ are the nonlinear and dispersion coefficients, respectively. Here and henceforth, subscripts $t$ and $x$ indicate derivatives with respect to the argument $t$ and $x$. We set $\beta=6$ and $\gamma_{3}=-1$ to obtain the focusing $\mathrm{KdV}$. We take the known hierarchy of rogue wave solutions of the mKdV [23] and apply a complex Miura transformation [26]

$$
q_{j}(x, t)=-p_{j}(x, t)^{2}+i\left(p_{j}\right)_{t}
$$

to them, where $j=1,2, \ldots$ is the order of the solution. Then $q(x, t)=q_{j}(x, t)$ is a solution of the complex $\mathrm{KdV}$,

$$
q_{x}(x, t)-6 q(x, t) q_{t}(x, t)+q_{t t t}(x, t)=0 .
$$

The transformation (2) differs from the ordinary Miura transformation in providing the complex function as a result. This function is the solution of $\mathrm{KdV}$ in standard form but the solutions are now complex. Direct substitution confirms that they are indeed complex solutions of the $\mathrm{KdV}$ equation.

\section{FIRST-ORDER RATIONAL SOLUTION}

The first-order rational solution of the $\mathrm{mKdV}$ can be written as [23]

$$
p_{1}=\frac{12 \gamma_{3}}{3 \gamma_{3}-2 \beta(t-\beta x)^{2}}-1,
$$

where velocity $v=\beta$. The solution resembles a soliton with a fixed velocity $v$. Thus, here, we have

$$
p_{1}=\frac{4}{4(t-6 x)^{2}+1}-1 \text {. }
$$

We use the above-mentioned complex Miura transformation for $j=1, q_{1}(x, t)=-p_{1}(x, t)^{2}+i p_{1}^{(0,1)}(x, t)$. We obtain

$$
q_{1}(x, t)=\frac{8}{(2 t-12 x+i)^{2}}-1,
$$

so the intensity is

$$
\left|q_{1}(x, t)\right|^{2}=16 \frac{5-4(t-6 x)^{2}}{\left[4(t-6 x)^{2}+1\right]^{2}}+1,
$$

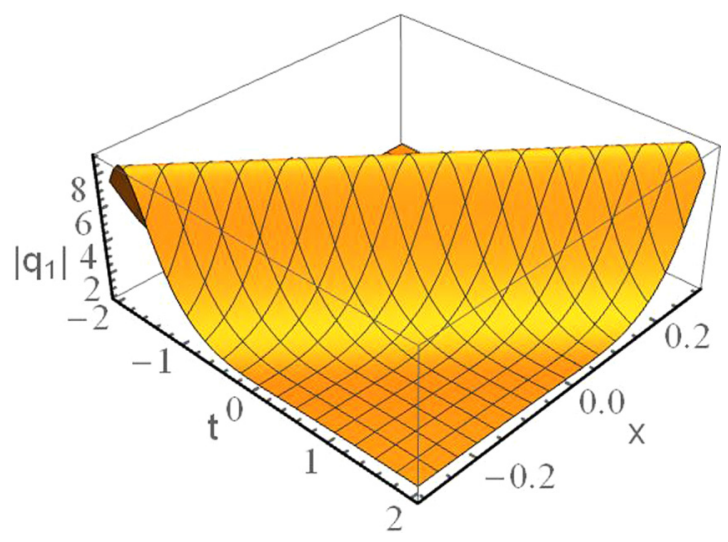

FIG. 1. First-order rational solution of complex $\mathrm{KdV},\left|q_{1}\right|$, given by Eq. (5). Here, $\gamma_{3}=-1$ and $\beta=6$. The maximal amplitude of the solution $\left|q_{1}\right|$ is 9 while the background level is 1 .

Hence we have

$$
q_{1}(x, t)+1=\frac{8}{F_{1}^{2}}=-2 \frac{\partial^{2}}{\partial t^{2}}\left(\log F_{1}\right),
$$

where $F_{1}=2 t-12 x+i$. For convenience, we set $X=12 x$ and $T=2 t$ throughout this Rapid Communication. Hence, $F_{1}=T-X+i$.

We plot the solution in Fig. 1. The maximal amplitude of the solution $\left|q_{1}\right|=9$ occurs along a straight line. The maximum is remarkably high in comparison to the maximum of the first-order NLSE and mKdV rogue waves that is 3 in each case. The background level here is -1 . This solution was obtained in Ref. [29], and clearly it was not related to rogue waves as the maximum is not a single peak. Rather than a rogue wave, it resembles a soliton with a nonzero velocity, although it is not the usual KdV soliton either. Plainly, it is not a "rogue wave," because the localization here is only in one dimension. Below, we will find that the higher-order solutions of the same hierarchy do have rogue wave features.

\section{SECOND-ORDER RATIONAL SOLUTION}

The second-order solution of the $\mathrm{mKdV}$ equation has been obtained in Ref. [23]. Using $\beta=6$ and $\gamma_{3}=-1$ to get coefficients $a=-2$ and $b=2$,

$$
p_{2}=\frac{12 G_{2}}{D_{2}}+1 \text {. }
$$

Here,

$$
\begin{aligned}
G_{2} & =3-\left[(T-X)^{3}+2(3 T-11 X)\right](T-X) \\
& =3-2\left[8(t-6 x)^{3}+12(t-22 x)\right](t-6 x)
\end{aligned}
$$

and

$$
\begin{aligned}
D_{2}= & 9+T^{6}-6 T^{5} X+3 T^{4}\left(5 X^{2}+1\right) \\
& +4 T^{3} X\left(1-5 X^{2}\right)+3 T^{2}\left[5\left(X^{2}-2\right) X^{2}+9\right] \\
& -6 T X\left(X^{4}-6 X^{2}+17\right)+X^{6}-13 X^{4}+139 X^{2} .
\end{aligned}
$$

We apply the complex Miura transformation to $p_{2}$. Remarkably, we can write $q_{2}(x, t)$ in terms of one function 

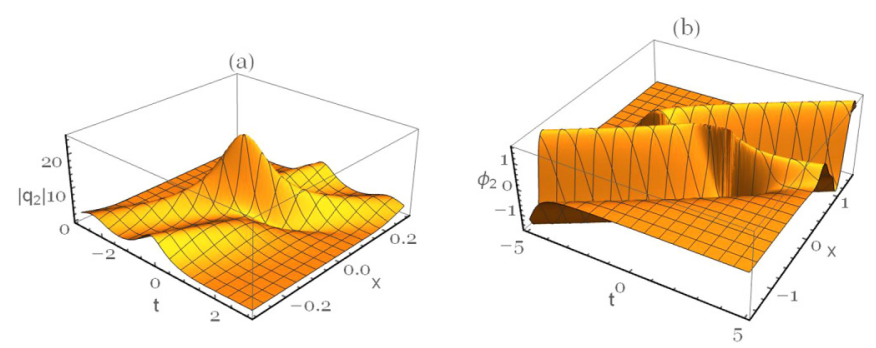

FIG. 2. (a) Second-order rational solution, $\left|q_{2}\right|$, given by Eq. (9) of complex KdV. Here, $\gamma_{3}=-1, \beta=6$. The maximum of $\left|q_{2}\right|$ is 25 and this occurs at the origin. (b) Phase $\phi_{2}$ of the same solution.

only,

$$
q_{2}(x, t)+1=-2 \frac{\partial^{2}}{\partial t^{2}}\left(\log F_{2}\right)
$$

where

$$
F_{2}=(T-X)^{3}+11 X-3 T-3 i\left[1+(T-X)^{2}\right],
$$

with $X=12 x$ and $T=2 t$, as above. We note that $\frac{\partial}{\partial t}\left(F_{2}\right)=$ $6\left(F_{1}^{*}\right)^{2}$, where ${ }^{*}$ indicates the complex conjugate. Thus,

$$
\begin{aligned}
F_{2}= & 2\left[4 t^{3}-72 t^{2} x+t\left(432 x^{2}-3\right)-864 x^{3}\right. \\
& +66 x]-3 i\left[4(t-6 x)^{2}+1\right] .
\end{aligned}
$$

Hence, $q_{2}(x, t)+1=24 \frac{N_{2}}{F_{2}^{2}}$, where

$$
\begin{aligned}
N_{2}= & T^{4}-4 T^{3}(X+i)+6 T^{2}(X+i)^{2} \\
& -4 T\left(X^{3}+3 i X^{2}+X-3 i\right) \\
& +\left(X^{2}+1\right)\left(9+4 i X+X^{2}\right) .
\end{aligned}
$$

The maximum of the solution Eq. (9) at the point $(0,0)$ is 25 . This is significantly higher than the maximum of the second-order NLSE rogue wave solution. The wave profile resembles a peak on top of a moving soliton. As the peak is higher than the soliton, the central bump can be interpreted as a rogue wave. The profile of the solution magnitude, Eq. (9), is shown in Fig. 2(a) for $\beta=6$. In Fig. 2(b), we show the phase, defined as $\phi_{2}=\arctan \left(\frac{\operatorname{Im}\left(q_{2}\right)}{\operatorname{Re}\left(q_{2}\right)}\right)$.

\section{THIRD-ORDER SOLUTION}

The general third-order rational solution can be obtained similarly,

$$
q_{3}(x, t)+1=-2 \frac{\partial^{2}}{\partial t^{2}}\left(\log F_{3}\right)
$$

where

$$
\begin{aligned}
F_{3}= & T^{6}-6 T^{5}(X-i)+15 T^{4}(X-i)^{2} \\
& -20 T^{3} X\left(X^{2}-3 i X-5\right)+15 T^{2}(X-i)\left(X^{3}\right. \\
& \left.-3 i X^{2}-11 X-3 i\right)-6 T\left(X^{5}-5 i X^{4}-30 X^{3}\right. \\
& \left.+40 i X^{2}+5 X-15 i\right)+X^{6}-6 i X^{5}-55 X^{4} \\
& +120 i X^{3}-245 X^{2}-450 i X-45,
\end{aligned}
$$

with $X=12 x$ and $T=2 t$.
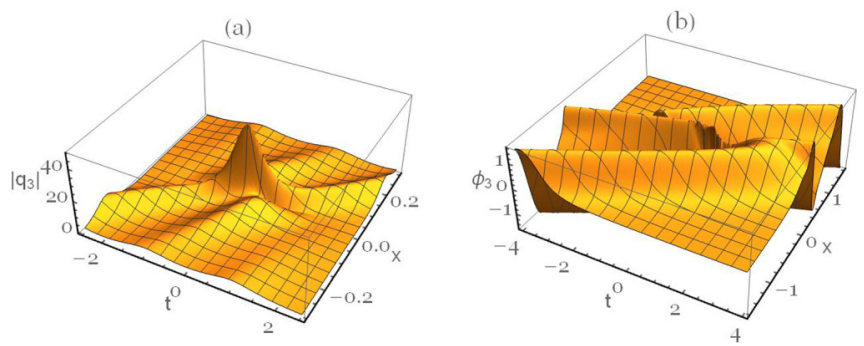

FIG. 3. (a) Third-order rational solution of complex $\mathrm{KdV},\left|q_{3}\right|$, given by Eq. (14). Here, $\gamma_{3}=-1, \beta=6$. The maximum is 49 and this occurs at the origin. (b) Phase $\phi_{3}$ of the same solution.

Hence, we can write this solution in the form

$$
q_{3}(x, t)=48 \frac{N_{3}}{F_{3}^{2}}-1 \text {, }
$$

where the explicit form of $N_{3}$ calculated from $F_{3}$ is given in the Appendix. The profile of the solution magnitude, Eq. (14), is shown in Fig. 3(a) for $\beta=6$. In Fig. 3(b), we show the phase, defined as $\phi_{3}=\arctan \left(\frac{\operatorname{Im}\left(q_{3}\right)}{\operatorname{Re}\left(q_{3}\right)}\right)$.

In Table I, we summarize the findings. The first column in the table shows the order of the solution while the second and third columns show the background and height involved in each $p(\mathrm{mKdV})$ solution. The fourth and fifth columns show the background and maximum amplitude involved in each $q$ $(\mathrm{KdV})$ solution. The last row shows the general expressions for the background and amplitude for any order $j$.

The maximum amplitude increases rapidly with the order $j$. This is the main characteristic of these solutions that allows us to claim that they describe rogue waves. Taking into account the fact that the background level has unit magnitude, the "amplification factor" can significantly exceed that for NLSE rogue waves. Our present results demonstrate, clearly, that shallow-water rogue waves can indeed be devastating.

\section{CONSERVED QUANTITIES}

The physical relevance of these solutions is further confirmed by the existence of conserved quantities. The scattering of multisolitons that are solutions of the $\mathrm{KdV}$ equation for the complex field has been considered earlier in Ref. [30]. Although the wave functions are complex, the quantities that correspond to mass, momentum, and energy are real. Clearly, they must be relevant to both solitons and rogue waves.

We start with the conservation of mass. We have, for any $j$, $q_{j}(x, t)+1=-2 \frac{\partial^{2}}{\partial t^{2}}\left(\log F_{j}\right)$. Consequently, the quantity

$$
\begin{aligned}
I_{j} & =\int_{-\infty}^{\infty}\left[q_{j}(x, t)+1\right] d t \\
& =-2\left[\frac{\partial}{\partial t}\left(\log F_{j}\right)\right]_{-\infty}^{\infty}=0
\end{aligned}
$$

is zero for all $x$, since $\frac{\partial}{\partial t}\left(\log F_{j}\right)=\frac{1}{F_{j}} \frac{\partial}{\partial t}\left(F_{j}\right)$ and this latter expression varies as $\approx 1 / t$ for large $|t|$. This proves that, indeed, the mass defined by the above expression is conserved.

Next, we define the momentum as $M_{j}=$ $\int_{-\infty}^{\infty}\left[q_{j}^{2}(x, t)-1\right] d t=\int_{-\infty}^{\infty}\left(q_{j}+1\right)^{2} d t . \quad$ These two expressions are equal due to Eq. (15). Then, $M_{j}=0$ for all $x$. 
TABLE I. Background level and the amplitude of rational solutions of order $j$ for mKdV (two left columns) and KdV (two right columns) equations.

\begin{tabular}{ccccc}
\hline \hline$j$ & Background level of $p$ & Height of $p$ & Background level of $q$ & Maximum of $\left|q_{j}\right|$ \\
\hline 1 & -1 & 3 & -1 & $3^{2}=9$ \\
2 & +1 & 5 & -1 & $5^{2}=25$ \\
$7^{2}=49$ & $(2 j+1)^{2}$ \\
3 & -1 & 7 & -1 & $(-1)$ \\
\end{tabular}

The energy is defined as $E_{j}=\int_{-\infty}^{\infty}\left[2\left(q_{j}+1\right)^{3}+\left(q_{j, t}\right)^{2}\right] d t$. For the first-order solution, $E_{1}=2048 \int_{-\infty}^{\infty}(2 t-12 x+$ $i)^{-6} d t$. It is easy to verify that $E_{j}=0$ for each order $j$.

\section{WATER SURFACE ELEVATION}

The next question is how to apply these results to finding the water surface elevation in a channel with constant depth. Rough estimates can be made on the basis of Levi's model [15], where the real part of the function $q(x, t)$ corresponds to the horizontal component of the fluid velocity along the channel while the imaginary part corresponds to the vertical component of the velocity, i.e., by the $t$ derivative of the matching $\mathrm{mKdV}$ solution $\left(p_{j}\right)_{t}$, as follows from Eq. (2). In this instance, the water elevation $H$ is roughly defined by the indefinite integral of the imaginary part of the complex field,

$$
H \approx \int \frac{\partial}{\partial t}\left[p_{j}(x, t)\right] d x+C .
$$

The integration constant $C$ is normally chosen in such a way as to keep the water level at zero around the rogue wave event. Figure 4 shows two plots that have been constructed this way. The one in Fig. 4(a) is for the second-order solution and the one in Fig. 4(b) is for the third-order solution. The amplitudes here are not as dramatic as for the modulus of the function $|q|$, but the rogue wave peak is certainly there. Interestingly, it is not located at the origin. More accurate estimates should be made using the full scale theory provided by the work of Levi [15]. The latter requires not only the dependent but also the independent variables to be complex.

\section{CONCLUSIONS}

To summarize, we have presented the lowest-order complex rational solutions of the $\mathrm{KdV}$ equation. These are the first three members of an infinite hierarchy of rogue wave
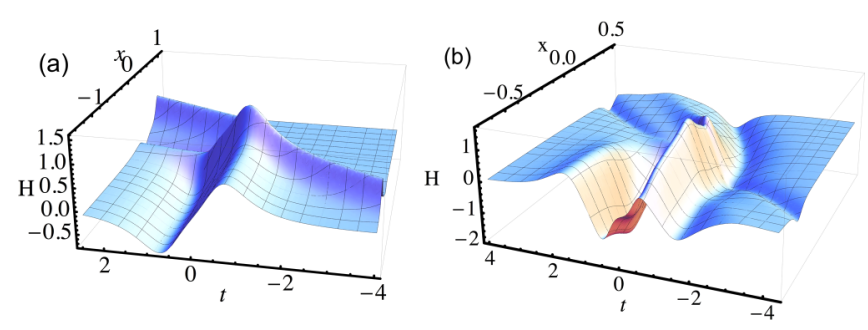

FIG. 4. Water level elevation for (a) second- and (b) third-order rational solutions of complex $\mathrm{KdV}$. Here, $\gamma_{3}=-1, \beta=6$. solutions. We used the complex Miura transformation to derive them. However, other methods such as the Darboux transformation or equivalent can also be used to obtain the whole hierarchy, as is often done for other integrable equations $[31,32]$. These solutions imply the existence of shallowwater rogue waves with amplitudes exceeding the soliton collision amplitudes of the real KdV equation. Namely, they are proportional to the square of the order of the solution [1]. Thus, a collision of solitons does not explain rogue waves. Indeed, the peak amplitudes at the point of soliton collision are too low to count them as rogue waves.

The significance of our findings is not restricted to water rogue waves. Our findings can be applied to optics, where the ideas of shallow-water rogue waves are also applicable [33]. They can occur in diffusion-controlled unidirectional crystal growth [34]. One- and two-soliton complex solutions of the $\mathrm{KdV}$ equation have been found and related to $\mathcal{P} \mathcal{T}$-symmetric systems [35,36], quantum-mechanical scattering matrices in a semiclassical approximation [30], and to a number of other physical systems where the $\mathrm{KdV}$ is the governing equation.

\section{ACKNOWLEDGMENT}

The authors acknowledge the support of the Australian Research Council (ARC).

\section{APPENDIX}

The expression in $N_{3}$ of Eq. (14) is given by

$$
\begin{aligned}
N_{3}= & T^{10}-10 T^{9}(X-i)+45 T^{8}(X-i)^{2} \\
& -120 T^{7}(X-i)^{3}+210 T^{6}(X-i)^{4} \\
& -36 T^{5}\left(7 X^{5}-35 i X^{4}-70 X^{3}+70 i X^{2}+35 X\right. \\
& +5 i)+30 T^{4}\left(7 X^{6}-42 i X^{5}-105 X^{4}+140 i X^{3}\right. \\
& \left.+185 X^{2}+110 i X+45\right)-120 T^{3}\left(X^{7}-7 i X^{6}\right. \\
& \left.-21 X^{5}+35 i X^{4}+115 X^{3}+15 i X^{2}+125 X-15 i\right) \\
& +45 T^{2}(X-i)^{2}\left(X^{6}-6 i X^{5}-15 X^{4}+20 i X^{3}\right. \\
& \left.+335 X^{2}+410 i X+15\right)-10 T\left(X^{9}-9 i X^{8}-36 X^{7}\right. \\
& +84 i X^{6}+1086 X^{5}-1830 i X^{4}-740 X^{3}-4380 i X^{2} \\
& -135 X+135 i)+X^{10}-10 i X^{9}-45 X^{8} \\
& +120 i X^{7}+2610 X^{6}-7020 i X^{5}-16250 X^{4} \\
& -13000 i X^{3}-17475 X^{2}-5850 i X-2025 .
\end{aligned}
$$


[1] T. Soomere, Eur. Phys. J.: Spec. Top. 185, 81 (2010).

[2] K. Dysthe, H. E. Krogstad, and P. Müller, Annu. Rev. Fluid Mech. 40, 287 (2008).

[3] A. Osborne, Nonlinear Ocean Waves and the Inverse Scattering Transform (Elsevier, Amsterdam, 2010).

[4] C. Kharif, E. Pelinovsky, and A. Slunyaev, Rogue Waves in the Ocean (Springer, Heidelberg, 2009).

[5] M. Onorato, S. Residori, U. Bortolozzo, A. Montina, and F. T. Arecchi, Phys. Rep. 528, 47 (2013).

[6] I. Nikolkina and I. Didenkulova, Nat. Hazards Earth Syst. Sci. 11, 2913 (2011).

[7] V. E. Zakharov, J. Appl. Mech. Tech. Phys. 9, 190 (1968).

[8] N. Akhmediev, A. Ankiewicz, and M. Taki, Phys. Lett. A 373, 675 (2009).

[9] V. Shrira and V. Geogjaev, J. Eng. Math. 67, 11 (2010).

[10] A. Chabchoub, N. P. Hoffmann, and N. Akhmediev, Phys. Rev. Lett. 106, 204502 (2011).

[11] Y. Kodama, J. Phys. A: Math. Theor. 43, 434004 (2010).

[12] D. J. Korteweg and G. De Vries, Philos. Mag. 39, 422 (1895).

[13] N. J. Zabusky and M. D. Kruskal, Phys. Rev. Lett. 15, 240 (1965).

[14] N. J. Zabusky and C. J. Galvin, J. Fluid Mech. 47, 811 (1971).

[15] D. Levi, Teor. Mat. Fiz. 99, 435 (1994) [Theor. Math. Phys. 99, 705 (1994).]

[16] H. I. Abdel-Gawad, M. Tantawy, and R. E. A. Elkhair, Waves Random Complex Media 26, 397 (2016),

[17] D. Levi and M. Sanielevici, Physica D 98, 510 (1996).

[18] A. R. Osborne and G. Boffetta, Phys. Fluids A 1, 1200 (1989).

[19] A. V. Slunyaev, Zh. Eksp. Teor. Fiz. 128, 1061 (2005) [JETP 101, 926 (2005)].
[20] T. P. Horikis and D. J. Frantzeskakis, Rom. J. Phys. 59, 195 (2014).

[21] G. Schneider, J Differ. Equations 147, 333 (1998).

[22] G. Schneider, Adv. Math. Phys. 2011, 854719 (2011).

[23] A. Ankiewicz and N. Akhmediev, Nonlinear Dyn. 91, 1931 (2018).

[24] R. M. Miura, J. Math. Phys. 9, 1202 (1968).

[25] R. M. Miura, C. S. Gardner, and M. D. Kruskal, J. Math. Phys. 9, 1204 (1968).

[26] B. Buti, N. N. Rao, and S. B. Khadkikar, Phys. Scr. 34, 729 (1986).

[27] Y. Zhang, Y.-n. Lv, L.-y. Ye, and H.-q. Zhao, Phys. Lett. A 367, 465 (2007).

[28] J.-M. Yuan and J. Wu, Discrete Contin. Dyn. Syst. B 5, 489 (2005).

[29] Y.-Y. Sun, J.-M. Yuan, and D.-J. Zhang, Commun. Theor. Phys. 61, 415 (2014).

[30] J. Cen, F. Correa, and A. Fring, J. Math. Phys. 58, 032901 (2017).

[31] F. Baronio, A. Degasperis, M. Conforti, and S. Wabnitz, Phys. Rev. Lett. 109, 044102 (2012).

[32] Y. Ohta and J. K. Yang, Phys. Rev. E 86, 036604 (2012).

[33] S. Wabnitz, C. Finot, J. Fatome, and G. Millot, Phys. Lett. A 337, 932 (2013).

[34] M. Kerszberg, Phys. Lett. A 105, 241 (1984).

[35] J. Cen and A. Fring, J. Phys. A: Math. Theor. 49, 365202 (2016).

[36] S. Modak, A. P. Singh, and P. K. Panigrahi, Eur. Phys. J. B 89, 149 (2016). 